\title{
Titration of Inspired Oxygen in Preterm Infants with Hypoxemic Respiratory Failure Using Near Infrared Spectroscopy and Pulse Oximetry: A New Approach
}

\author{
Yasser Elsayed ${ }^{1}$ and Shyamala Dakshinamurti ${ }^{2}$ \\ ${ }^{1}$ University of Manitoba Faculty of Health Sciences \\ ${ }^{2}$ University of Manitoba
}

May 15, 2021

\begin{abstract}
Background: Titration of inspired oxygen is a challenge in preterm infants with hypoxemic respiratory failure (HRF). Monitoring of brain oxygen by near infrared spectroscopy (NIRS) has been proven to minimize the burden of hyperoxia and hypoxemia, with better understanding of cerebral autoregulation (CAR). integrating NIRS and pulse oximetry (SpO2) for titrating inspired oxygen is a novel approach. Methods: We aimed to study the impact of integrated monitoring of oxygen saturation by SpO2 and cerebral regional tissue oxygen (crRTO) by NIRS during oxygen reduction test (ORT) on reducing oxygen requirement in preterm infants with HRF. The correlation between $\mathrm{SpO} 2$ with crRTO, and fractional oxygen extraction (FOE) was assessed, concordance levels $(r>0.5)$ were determined during the assessment period, and was considered as a sign of impaired autoregulation. The primary outcome was the achievement of significantly lower FiO2 at 72 hours after start of the integrated monitoring. Results: Total of 38 preterm infants were included, 27 had normal cerebral autoregulation (CAR), (group 1) in whom SpO2 was poorly correlating with cerebral regional tissue oxygen $(\mathrm{crRTO})$ with $(\mathrm{r}<0.5)$ and had significantly greater percentage of reduction below baseline in FiO2 (Mean:34\%). Eleven infants had impaired CAR (group 2) with SpO2 significantly correlating with crRTO $(\mathrm{r}>0.5)$ and had a linear trend of FOE inverse to $\mathrm{SpO} 2$ and crRTO; this was considered as an arterial saturation dependent oxygen delivery (SadDO2). Conclusion: Integrated monitoring of preterm infants by SpO2 and crRTO was associated with easier weaning of oxygen with less burden of both hyperoxia and hypoxemia.
\end{abstract}

\section{Introduction:}

Since many preterm infants have impaired lung function, oxygen is one of the most common therapies in neonatal medicine(1). However, preterm infants are also particularly sensitive to the harmful effects of oxygen $(2,3)$. Highly reactive free oxygen radicals cause cellular injury(4). Preterm infants are not adequately protected from such direct biochemical oxidative stress $(3,5-7)$. Oxygen saturation, blood flow and hemoglobin are the three main components of oxygen delivery, and relying solely on arterial oxygen saturation may not adequately monitor tissue exposure to either hypoxia or hyperoxia(8-10). What is the optimal target arterial oxygen saturation as measured by pulse oximetry $\left(\mathrm{SpO}_{2}\right)$ in infants with hypoxemic respiratory failure (HRF) in the neonatal intensive care unit (NICU), is still an unanswered question $(3,5,11)$. Despite many recent large-scale studies, it is unclear if either low or high target oxygen saturation is safe, provides adequate organ oxygen delivery, and at the same time limits oxygen toxicity and accumulation of reactive oxygen species(3). Accepting lower oxygen saturation targets in infants with acute lung injury may limit toxic oxygen exposure, and enable weaning of inspired oxygen over a shorter period of time(5). However the outcome using this approach is dependent upon the ability of the organs to autoregulate blood flow $(12,13)$. Cerebral autoregulation is defined as the interaction between locally released nitric oxide from red blood cells (RBCs), and the vasodilator ability of the arterioles in tissues, in order to protect oxygen delivery during 
active tissue metabolism and maintain oxygen saturation of the brain during hypoxemic episodes, in a setting of acceptable hemoglobin, carbon dioxide $\left(\mathrm{CO}_{2}\right)$ and normal cerebral blood flow $(12,14,15)$. In preterm infants, we do not know if CAR can adequately protect the brain during even brief periods of desaturation or intermittent hypoxemia(16). Our group has developed an integrated approach set-up to provide us with the novel ability to longitudinally measure cerebral autoregulation in real time, in infants undergoing cerebral oxygen and hemodynamics monitoring(17-19). Although it is standard of practice in modern neonatal intensive care units to monitor $\mathrm{SpO}_{2}$ via pulse oximetry, a limited number of units (including our unit) use near infrared spectroscopy (NIRS) to monitor tissue oxygenation, and there is insufficient evidence that either mild desaturations or hypoxemia impact cerebral autoregulation. Understanding the temporal relationship between desaturation and cerebral blood flow autoregulation should lead to individualized safer weaning of oxygen while maintaining the integrity of $\operatorname{CAR}(18,20,21)$.

We hypothesized that CAR can compensate for short periods of hypoxemia or desaturation in preterm infants with normal hemodynamics and hemoglobin, thus maintaining brain oxygen tension, and this compensatory role of CAR can be clinically recognized as low $\mathrm{SpO} 2$ below target limits but crRTO is maintained without change.

\section{Methods:}

This was a retrospective study conducted in two level III NICUs in Winnipeg, Manitoba, Canada from April 2015 to July 2020. We included infants with HRF who underwent a CAR assessment with integrated monitoring by $\mathrm{SpO}_{2}$ and crRTO by NIRS using the following scheme: 1) a baseline assessment of mean $\mathrm{SpO}_{2}$, and $\mathrm{FiO}_{2}$ for the previous 24 hours before CAR assessment, together with baseline hemoglobin (Hb) and blood gases; 2) assessment of hemodynamics with a predefined targeted neonatal echocardiography protocol, excluding infants with pulmonary hypertension, significant PDA and circulatory shock; 3) a CAR assessment test, which is stepwise reduction of $\mathrm{FiO}_{2}$ by 0.02 every 2 minutes with continuous monitoring of $\mathrm{SpO}_{2}$ by pulse oximetry, and crRTO by NIRS. The infant should be quiet without significant handling for at least 30 minutes before the test, $\mathrm{SpO}_{2}$ should be between 90 to $95 \%$ before starting, and must be maintained within the acceptable range as per unit protocol $(86 \%$ to $94 \%)$ during the test, or the test was discontinued. Following the CAR test, routine saturation monitoring including $\mathrm{SpO}_{2}$ and NIRS was continued with titration of $\mathrm{FiO} 2$ with maintaining $\mathrm{SpO} 2$ as above and crRTO between 60 to $80 \%$ for 72 hours or until weaning $\mathrm{FiO} 2$ to $<0.3$. Institutional ethics board approval was obtained before commencing this study, figure 1 is an algorithm clarifies the steps of ORT and assessment of CAR.

\section{Definitions of terms used in this study:}

Hypoxemic respiratory failure in premature infants:

Preterm infant $<28$ weeks gestational age and beyond the postnatal transition, who is on invasive respiratory support with increasing $\mathrm{FiO}_{2}$ for $>24$ hours before assessment.

Fluctuation of arterial oxygen saturation:

Swinging of $\mathrm{SpO}_{2}$ above or below the acceptable target saturation (86-94\% for our unit)

Hypoxemia:

Arterial oxygen saturation $<80 \%(3,5,11)$

Mild desaturation:

Arterial oxygen saturation $<85 \%$ and $>80 \%$

\section{Monitoring of oxygenation:}

Arterial Oxygen saturation $\left(\mathrm{SpO}_{2}\right)$ was monitored using the Masimo Rad 7 Massimoß) (Massimo Corporation, Irvine, California) pulse oximeter; Cerebral regional tissue oxygen (crRTO) was measured by NIRS (FORE-SIGHT ${ }^{\circledR}$ Absolute Tissue Monitor, Casmed®, Branford, CT). The sensor was applied to the frontal 
area of the head; fractional oxygen extraction (FOE)(22) was defined physiologically as the ratio between oxygen delivery and regional oxygen consumption using $\operatorname{NIRS}(23),(24)$, calculated as $\mathrm{FOE}=\left(\mathrm{SpO}_{2}-\mathrm{RTO}\right)$ $/ \mathrm{SpO}_{2}$ (all expressed as fractions). The Oxygen saturation index (OSI)(25) $=\mathrm{MAP} \times \mathrm{FiO}_{2} \times 100 / \mathrm{SpO}_{2}$ was used as a marker of the severity of $\mathrm{HRF}$ in each studied patient, and $\mathrm{SpO} 2$ : $\mathrm{FiO} 2$ ratio was corrected with crRTO.

\section{Data analysis and assessment of CAR:}

The $\mathrm{SpO}_{2}$, heart rate (HR) and crRTO data were archived in the memory of the pulse oximeter and NIRS machines as a value recorded every 2 seconds, and transferred after the CAR test for further off line analysis. $\mathrm{FiO}_{2}$ was captured from records of mechanical ventilation. All data were studied using SPSS program to test the correlation of $\mathrm{SpO}_{2}$ with each of crRTO, $\mathrm{FiO}_{2}$, calculated FOE, and HR. The correlation between $\mathrm{SpO}_{2}$ and crRTO was also assessed during the most significant hypoxemic episodes, measured at rest without handling, during the monitoring period. Significant concordance $(r>0.5)$ is seen when oxygen delivery becomes dependent on arterial saturation, due to impaired CAR. The end point of the integrated monitoring was 72 hours after the CAR assessment test.

\section{Titration of $\mathrm{FiO}_{2}$ after CAR test:}

Titration of inspired oxygen was continued as per unit protocol, relying on combined monitoring of crRTO (targeted between 60 to 80\%) and $\mathrm{SpO} 2$ (targeted between $86 \%$ to $94 \%$ ), in those infants with maintained $\mathrm{CAR}$, and who had successful weaning of supplemental oxygen more than $20 \%$ below the starting $\mathrm{FiO}_{2}$ by the end of the CAR test. $\mathrm{FiO}_{2}$ could be weaned stepwise by $0.02 \mathrm{FiO}_{2}$ if $\operatorname{crRTO}$ was $>80 \%$, even when $\mathrm{SpO} 2$ is within acceptable target saturation(20). Mild desaturations and short self-resolved hypoxemic episodes were observed without intervention as long as crRTO was maintained within target limits.

\section{Statistical Analysis:}

SPSS v.24 (SPSS, Chicago, IL, USA) was used to perform the statistical analysis. Data presented as median with interquartile range or frequencies. Comparisons between groups analyzed by Mann- Whitney U-test; $p$ $<0.05$ considered significant. Pearson correlation was used to correlate between SpO2 and other variables, correlation was considered significant if $r>0.5$. GraphPad was used to instruct the correlation graph.

\section{Results:}

A total of 38 infants who had significant HRF were enrolled in the study. All of them had developed chronic lung disease, defined as requirement of respiratory support at postmenstrual age of 36 weeks; but all subsequently survived until discharge. None of the studied infants had intraventricular hemorrhage > grade II or were diagnosed with hydrocephalus. No infants had suspected or culture proven sepsis during the period under study. The assessed infants were at median inter quartile range (IQR) gestation (GA) and birth weight $(\mathrm{BW})$ of 26 weeks $(25,27)$ and $725 \mathrm{~g}(737,842)$ respectively. The patterns of response to CAR assessment were used to separate the studied infants into two groups: those with normal CAR (group 1) and those with impaired CAR (group 2). Twenty-seven infants tolerated the CAR assessment test (group 1) with median (IQR) duration of 12 minutes $(9,15$ minutes). Eleven infants did not tolerate CAR assessment (group 2) with desaturation on SpO2, associated with low crRTO on NIRS after the first few attempts of $\mathrm{FiO}_{2}$ reduction. Table 1 shows the basic clinical and hemodynamic parameters including $\mathrm{Hb}$, carbon dioxide $\left(\mathrm{CO}_{2}\right)$ and left heart cardiac output (LVO) which are the main confounders affecting oxygen delivery. There was no statistical difference between both groups regarding all basic clinical and hemodynamic parameters, other than infants in group 2 were less mature in gestational age, 4 infants from group 2 discharged home on oxygen and non from group 1. Table 2 shows the oxygen indices before and at the end of CAR assessment test. All infants in group 1 achieved a lower $\mathrm{FiO}_{2}$ after CAR assessment compared to their baseline; and all had significantly lower $\mathrm{FiO}_{2}$ (both as percent of reduction and as an absolute fraction; Mean percent of reduction :34\% and FiO2: 0.5) compared to group 2 (Mean percent of reduction of $5.3 \%$ and $\mathrm{FiO} 2$ of 0.63 ), calculated after CAR test and at 72 hours of integrated monitoring by pulse oximetry and NIRS. Group 1 had significantly higher crRTO and lower FOE (Mean of $71 \%$ and FOE of 0.13 ) compared to group 2 (Mean 
of $62 \%$ and FOE of 0.3$)$. There was no significant difference between both groups in OSI before and after CAR test, figure 2 shows the comparison between oxygen indices before and after ORT, group 1 (figure 2A) had significantly lower FiO2 at the end of ORT, and group 2 (figure 2B) had significantly lower crRTO and higher FOE at the end of ORT.

Phenotype of infants with intact CAR during assessment (group1):

Figure 3-A1 is a graphical representation of the $\mathrm{FiO}_{2}, \mathrm{SpO}_{2}$ and $\mathrm{HR}$ trends during CAR assessment test in one of the cases from group 1 . During stepwise reduction of $\mathrm{FiO}_{2}$ from 0.57 to 0.38 , the $\mathrm{SpO}_{2}$ was maintained (figure 3-A2). A self-resolved hypoxemic episode resulted in cessation of the test at 12 minutes. Figure 3-a (B) shows that despite $\mathrm{SpO}_{2}$ fluctuation, crRTO was maintained linearly; but FOE was concordant linearly with $\mathrm{SpO}_{2}$. Table 3 shows the poor positive correlation of $\mathrm{SpO}_{2}$ with crRTO and with $\mathrm{FiO}_{2}$, and poor negative correlation of $\mathrm{SpO} 2$ with $\mathrm{HR}(r<0.5)$. The correlation between $\mathrm{SpO}_{2}$ and FOE was strong in infants of this group $(r>0.5)$, and there was a poor correlation between $\mathrm{SpO} 2$ and crRTO during the significant hypoxemic episode.

Phenotype of infants with impaired CAR during assessment (group 2):

Figure 3-B shows discontinuation of $\mathrm{CAR}$ assessment after a few steps of $\mathrm{FiO}_{2}$ reduction due to significant fluctuation of $\mathrm{SpO}_{2}$ which was associated with bradycardias (figure 3-B1). Figure 3-B2 shows the linear concordance between $\mathrm{SpO}_{2}$ and crRTO. FOE was negatively correlated with both $\mathrm{SpO} 2$ and crRTO. Table 3 shows a strong positive correlation between $\mathrm{SpO} 2$ and crRTO, $\mathrm{HR}$, and $\mathrm{FiO} 2$, and strong negative correlation of $\mathrm{SpO} 2$ with FOE, and there was a significant positive correlation between $\mathrm{SpO} 2$ and crRTO during a significant hypoxemic episode.

\section{Discussion:}

Understanding the capacity of critical body organs to compensate for short periods of hypoxemia is important to individualize the acceptable $\mathrm{SpO}_{2}$ and crRTO for sick preterm infants. The degree of this compensation is largely dependent upon the ability of the cerebral vasculature to autoregulate blood flow and thus buffer brain oxygenation, and there is a gap in literature regarding mechanisms of compensation in infants with $\operatorname{HRF}(12,13)$. In this study we assessed patterns of CAR in preterm infants with HRF through graded stepwise reduction of $\mathrm{FiO}_{2}$, aiming to test the concordance between $\mathrm{SpO}_{2}$ and crRTO which represents integrity of autoregulation(26-28). Monitoring oxygen by pulse oximetry has several limitations, including different calibration between different devices, and the $\mathrm{SpO}_{2}$ on the monitor is a calibrated mean between 2 standard deviations, this might increase the chances of hyperoxia with higher delivered $\mathrm{FiO}_{2}(29,30)$. Autoregulatory mechanism is induced by a local vasodilator effect through local release of nitric oxide from red blood cells when there is decrease of oxygen delivery, and augments blood flow to buffer low delivery of oxygen; this serves as the initial brain rescue step in hypoxemia $(13,31)$. In some preterm infant this CAR might be impaired as a consequence of prematurity or severity of sickness, and it is almost absent for early postnatal transition period in very premature infants and increased oxygen extraction is the main compensatory mechanism with impaired or compromised autoregulation $(32,33)$. Infants with intact CAR (group 1) had low FOE, reasoning in reverse, we infer that maintenance of a normal crRTO and a low FOE signifies that CAR is still able to compensate for desaturations. Where FOE maintained or decreases as $\mathrm{SpO}_{2}$ decreases (as in figure 1B), it means that the brain tissue does not need to increase oxygen extraction to compensate for the decreased hemoglobin oxygen saturation, as CAR is compensating by increasing the volume of brain blood flow(20). In infants with impaired CAR (group 2) linear fluctuations of both $\mathrm{SpO} 2$ and crRTO trigger mirror image fluctuations of FOE, and increased FOE will immediately maintain cerebral oxygen demands (figure 2B), while $\mathrm{SpO}_{2}$, crRTO and HR are positively correlated. This concordance may represent sensitivity of SA node and chemoreceptors to brief hypoxemic episodes. We speculate that mild tachycardia during desaturations signifies intact autoregulation, but bradycardia represents failure of the sinoatrial node to continue firing at a physiologic rate due to energy compromise secondary to hypoxia. The strong positive correlation between $\mathrm{SpO}_{2}$ and $\mathrm{HR}$ in infants in group 2 signifies impaired CAR at the SA node level resulting in frequent bradycardia, which would also contributing to compromise of cerebral blood flow and oxygen delivery(16). 
Understanding the safe ranges of $\mathrm{FiO}_{2}$ at which $\mathrm{SpO}_{2}$ and crRTO are acceptable is considered helpful to individualize titration of inspired oxygen, aiming to avoid either cerebral hypoxia or hyperoxia(29). We utilized this approach to titrate $\mathrm{FiO}_{2}$ only in group 1 infants with intact $\mathrm{CAR}$, in whom we were able to wean $\mathrm{FiO}_{2}$ significantly and this is after teaching the nurses on how to wean oxygen according to both $\mathrm{SpO} 2$ and crRTO simultaneously. This oxygen titration approach was avoided in infants with a group 2 pattern of impaired autoregulation and arterial saturation-dependent oxygen delivery, this new physiologic approach was approved by our patient care committee as a standard of care.

This is the first study to our knowledge reporting CAR in preterm infants with HRF. The strength of our study is the development of a practical bedside application with direct clinically relevance to management of HRF. The main limitation is the retrospective design of the study, absence of long term follow up, and the small sample size, although this report can be considered as a quality assessment of the approved patient care protocol. The methods used here also cannot distinguish a FOE decrease due to a primary impairment of tissue oxygen utilization; however, infants studied were medically stable and non-septic, rendering toxic impairment of oxygen uptake unlikely.

\section{Conclusion:}

Identification of infants with intact CAR by assessment of cerebral oxygenation during weaning of supplemental oxygen resulted in easier titration of $\mathrm{FiO}_{2}$. Further studies are needed to confirm the underlying capacity of the preterm infants to compensate for hypoxemia, and to examine the impact of CAR assessment on long-term outcome.

Authors contribution:

Both authors made a substantial contribution to the manuscript and the design of the work: YE was the principal investigator and collected the data, SD helped in data analysis and in writing the manuscript.

Compliance with ethical statement:

Conflict of interest: The authors declare that they have no conflict of interest

Funding: there is no funding source

Ethical approval: The local ethical committee has approved to publish data.

References:

1. van Zanten H a., Tan RNGB, van den Hoogen A, Lopriore E, te Pas AB. Compliance in oxygen saturation targeting in preterm infants: a systematic review. Eur J Pediatr [Internet]. 2015; Available from: http://link.springer.com/10.1007/s00431-015-2643-0

2. The BOOST-II Australia and United Kingdom Collaborative Groups. Outcomes of Two Trials of OxygenSaturation Targets in Preterm Infants. N Engl J Med [Internet]. 2016;160210140037001. Available from: http://www.nejm.org/doi/abs/10.1056/NEJMoa1514212

3. Saugstad OD, Aune D. Optimal oxygenation of extremely low birth weight infants: a meta-analysis and systematic review of the oxygen saturation target studies. Neonatology [Internet]. 2014;105:55-63. Available from: http://www.ncbi.nlm.nih.gov/pubmed/24247112

4. Killilea DW, Hester R, Balczon R, Babal P, Gillespie MN. Free radical production in hypoxic pulmonary artery smooth muscle cells. Am J Physiol Lung Cell Mol Physiol [Internet]. 2000 Aug;279(2):L408-12. Available from: http://www.ncbi.nlm.nih.gov/pubmed/10926565

5. Schmidt B, Whyte RK, Asztalos E V, Moddemann D, Poets C, Rabi Y, et al. Effects of targeting higher vs lower arterial oxygen saturations on death or disability in extremely preterm infants: a randomized clinical trial. Jama [Internet]. 2013;309(20):2111-20. Available from: http://www.ncbi.nlm.nih.gov/pubmed/23644995 
6. Finer N, Saugstad O, Vento M, Barrington K, Davis P, Duara S, et al. Use of oxygen for resuscitation of the extremely low birth weight infant. Pediatrics. 2010;125(2):389-91.

7. Kenosi M, O'Toole JM, Livingston V, Hawkes GA, Boylan GB, O'Halloran KD, et al. Effects of Fractional Inspired Oxygen on Cerebral Oxygenation in Preterm Infants following Delivery. J Pediatr [Internet]. 2015;167(5):1007-1012.e1. Available from: http://dx.doi.org/10.1016/j.jpeds.2015.07.063

8. Schwartz S, Frantz RA, Shoemaker WC. Sequential hemodynamic and oxygen transport responses in hypovolemia, anemia, and hypoxia. Am J Physiol [Internet]. 1981;241(6):H864-71. Available from: http://www.ncbi.nlm.nih.gov/pubmed/7325255

9. Sun L, Macgowan CK, Sled JG, Yoo SJ, Manlhiot C, Porayette P, et al. Reduced fetal cerebral oxygen consumption is associated with smaller brain size in fetuses with congenital heart disease. Circulation. 2015;131(15):1313-23.

10. Wolff CB. Normal cardiac output, oxygen delivery and oxygen extraction. Adv Exp Med Biol. 2008;599:169-82.

11. Askie LM, Henderson-Smart DJ. Restricted versus liberal oxygen exposure for preventing morbidity and mortality in preterm or low birth weight infants.update of Cochrane Database Syst Rev. 2000;(2):CD001077; PMID: 10796409. Cochrane Database Syst Rev. 2009;1(3):CD001077.

12. Greisen G, Sci M. Cerebral Autoregulation in Preterm Infants. How to Measure It-And Why Care? J Pediatr [Internet]. 2014;165(5):885-6. Available from: http://dx.doi.org/10.1016/j.jpeds.2014.07.031

13. Vesoulis Z a., Liao SM, Trivedi SB, El Ters N, Mathur AM. A Novel Method for Assessing Cerebral Autoregulation in Preterm Infants Using Transfer Function Analysis. Pediatr Res [Internet]. 2015;(November). Available from: http://www.nature.com/doifinder/10.1038/pr.2015.238

14. Chock VY, Ramamoorthy C, Van Meurs KP. Cerebral Autoregulation in Neonates with a Hemodynamically Significant Patent Ductus Arteriosus. J Pediatr [Internet]. 2012 Jan [cited 2012 May 5];160(6):936-42. Available from: http://linkinghub.elsevier.com/retrieve/pii/S0022347611012285

15. Riera J, Caba F, Serrano J, Bravo C, Laura S. New Time-Frequency Method for Cerebral Autoregulation in Newborns: Predictive Capacity for Clinical Outcomes. j Pediatr.

16. Poets CF, Roberts RS, Schmidt B, Whyte RK, Asztalos E V., Bader D, et al. Association Between Intermittent Hypoxemia or Bradycardia and Late Death or Disability in Extremely Preterm Infants. Jama [Internet]. 2015;314(6):595. Available from: http://jama.jamanetwork.com/article.aspx?doi=10.1001/jama.2015.8841

17. Ali YH, Elsayed Y, Srinivasan G, Seshia MK, Al-Ethawi Y, Baier R. The impact of different modes of noninvasive ventilation on hemodynamics and regional cerebral and splanchnic oximetry in the premature newborn. $J$ Clin Neonatol [Internet]. 2016;5(3):179. Available from: http://www.jcnonweb.com/text.asp?2016/5/3/179/191256

18. Elsayed YN, Amer R, Seshia MM. The impact of integrated evaluation of hemodynamics using targeted neonatal echocardiography with indices of tissue oxygenation: a new approach. J Perinatol. 2017;

19. Amer R, Kalash R, Seshia MM, Elsayed YN. The Impact of Integrated Evaluation of Hemodynamics on Management of Preterm Infants with Late-Onset Compromised Systemic Circulation. Am J Perinatol. 2017;

20. Elsayed YN, Fraser D. Integrated evaluation of neonatal hemodynamics, part 2: Systematic bedside assessment. Neonatal Netw. 2016;35(4).

21. Elsayed YN, Fraser D. Integrated evaluation of neonatal hemodynamics program optimizing organ perfusion and performance in critically ill neonates, part 1: Understanding physiology of neonatal hemodynamics. Neonatal Netw. 2016;35(3). 
22. Petrova A, Mehta R. Near-infrared spectroscopy in the detection of regional tissue oxygenation during hypoxic events in preterm infants undergoing critical care. Pediatr Crit Care Med. 2006;7(5):449-54.

23. Pichler G, Binder C, Avian A, Beckenbach E, Schmölzer GM, Urlesberger B. Reference ranges for regional cerebral tissue oxygen saturation and fractional oxygen extraction in neonates during immediate transition after birth. J Pediatr. 2013;163(13228):1558-63.

24. Greisen G, Leung T, Wolf M. Has the time come to use near-infrared spectroscopy as a routine clinical tool in preterm infants undergoing intensive care? Philos Trans R Soc A Math Phys Eng Sci. 2011;369:4440-51.

25. Rawat M, Chandrasekharan PK, Williams A, Gugino S, Koenigsknecht C, Swartz D, et al. Oxygen saturation index and severity of hypoxic respiratory failure. Neonatology. 2015;107(3):161-6.

26. Alderliesten T, Lemmers PMA, Smarius JJM, Baerts W, Bel F Van. Cerebral Oxygenation, Extraction, and Autoregulation in Very Preterm Infants Who Develop Peri-Intraventricular Hemorrhage. 2013;

27. Chock VY, Kwon SH, Ambalavanan N, Batton B, Nelin LD, Chalak LF, et al. Cerebral Oxygenation and Autoregulation in Preterm Infants (Early NIRS Study). J Pediatr. 2020;227:94-100.e1.

28. Vesoulis Z a, Lust CE, Liao SM, Trivedi SB, Mathur a M. Early hyperoxia burden detected by cerebral near-infrared spectroscopy is superior to pulse oximetry for prediction of severe retinopathy of prematurity. J Perinatol [Internet]. 2016;36(April):1-6. Available from: http://www.ncbi.nlm.nih.gov/pubmed/27559717

29. Lakshminrusimha S, Manja V, Mathew B, Suresh GK. Oxygen targeting in preterm infants: a physiological interpretation. J Perinatol [Internet]. 2014;35(August):8-15. Available from: http://www.nature.com/doifinder/10.1038/jp.2014.199

30. Lakshminrusimha S, Konduri GG, Steinhorn RH. Considerations in the management of hypoxemic respiratory failure and persistent pulmonary hypertension in term and late preterm neonates. J Perinatol [Internet]. 2016;36(s2):S12-9. Available from: http://www.nature.com/doifinder/10.1038/jp.2016.44

31. Wardle SP, Yoxall CW, Weindling a M. Determinants of cerebral fractional oxygen extraction using near infrared spectroscopy in preterm neonates. J Cereb Blood Flow Metab. 2000;20(2):272-9.

32. Noori S, McCoy M, Anderson MP, Ramji F, Seri I. Changes in cardiac function and cerebral blood flow in relation to peri/intraventricular hemorrhage in extremely preterm infants. J Pediatr [Internet]. 2014;164(2):264-70.e1-3. Available from: http://www.ncbi.nlm.nih.gov/pubmed/24183212

33. Takami T, Sunohara D, Kondo A, Mizukaki N, Suganami Y, Takei Y, et al. Changes in cerebral perfusion in extremely LBW infants during the first $72 \mathrm{~h}$ after birth. Pediatr Res. 2010;68(5):435-9.

\section{Hosted file}

table 1.pdf available at https://authorea.com/users/413946/articles/522125-titrationof-inspired-oxygen-in-preterm-infants-with-hypoxemic-respiratory-failure-using-nearinfrared-spectroscopy-and-pulse-oximetry-a-new-approach

\section{Hosted file}

table 2.pdf available at https://authorea.com/users/413946/articles/522125-titrationof-inspired-oxygen-in-preterm-infants-with-hypoxemic-respiratory-failure-using-nearinfrared-spectroscopy-and-pulse-oximetry-a-new-approach

\section{Hosted file}

table 3.pdf available at https://authorea.com/users/413946/articles/522125-titrationof-inspired-oxygen-in-preterm-infants-with-hypoxemic-respiratory-failure-using-nearinfrared-spectroscopy-and-pulse-oximetry-a-new-approach 

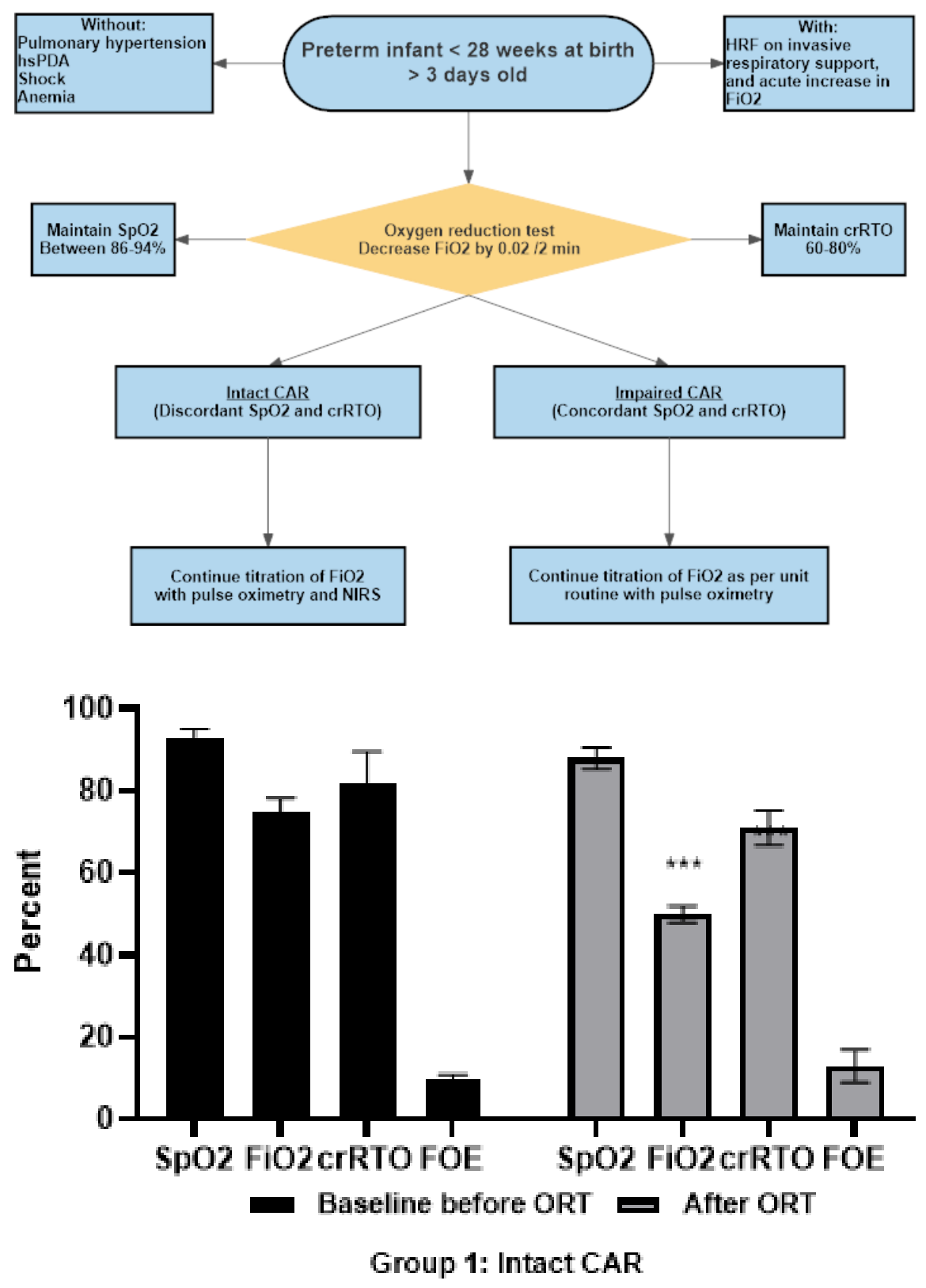


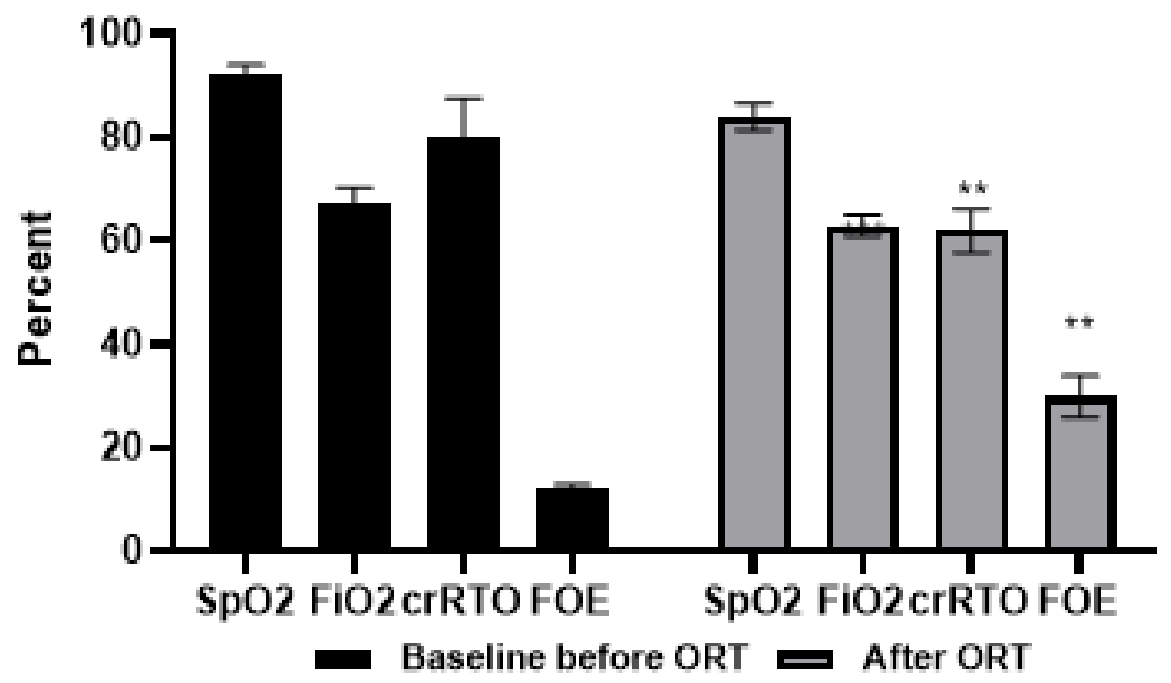

Group 2: Impaired CAR

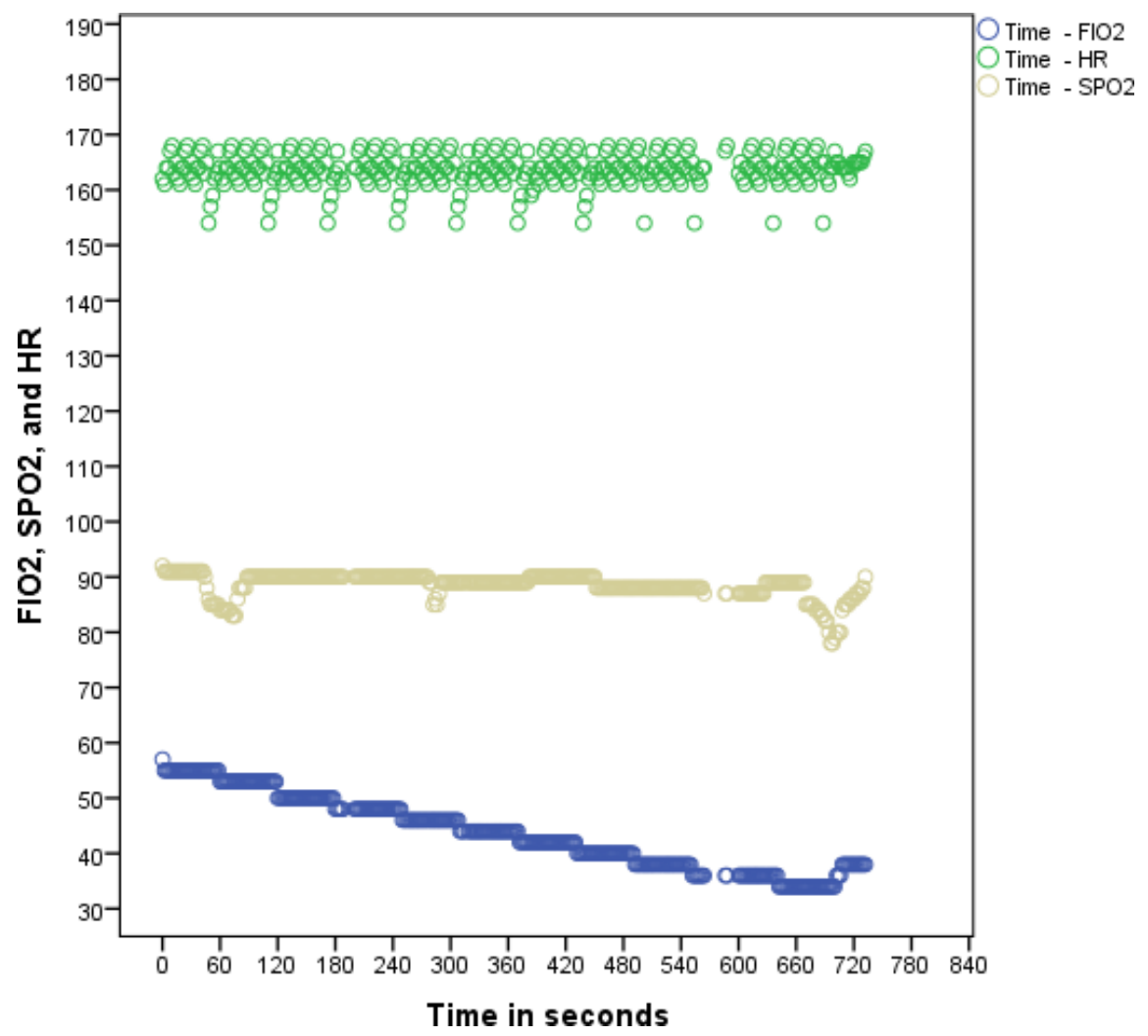



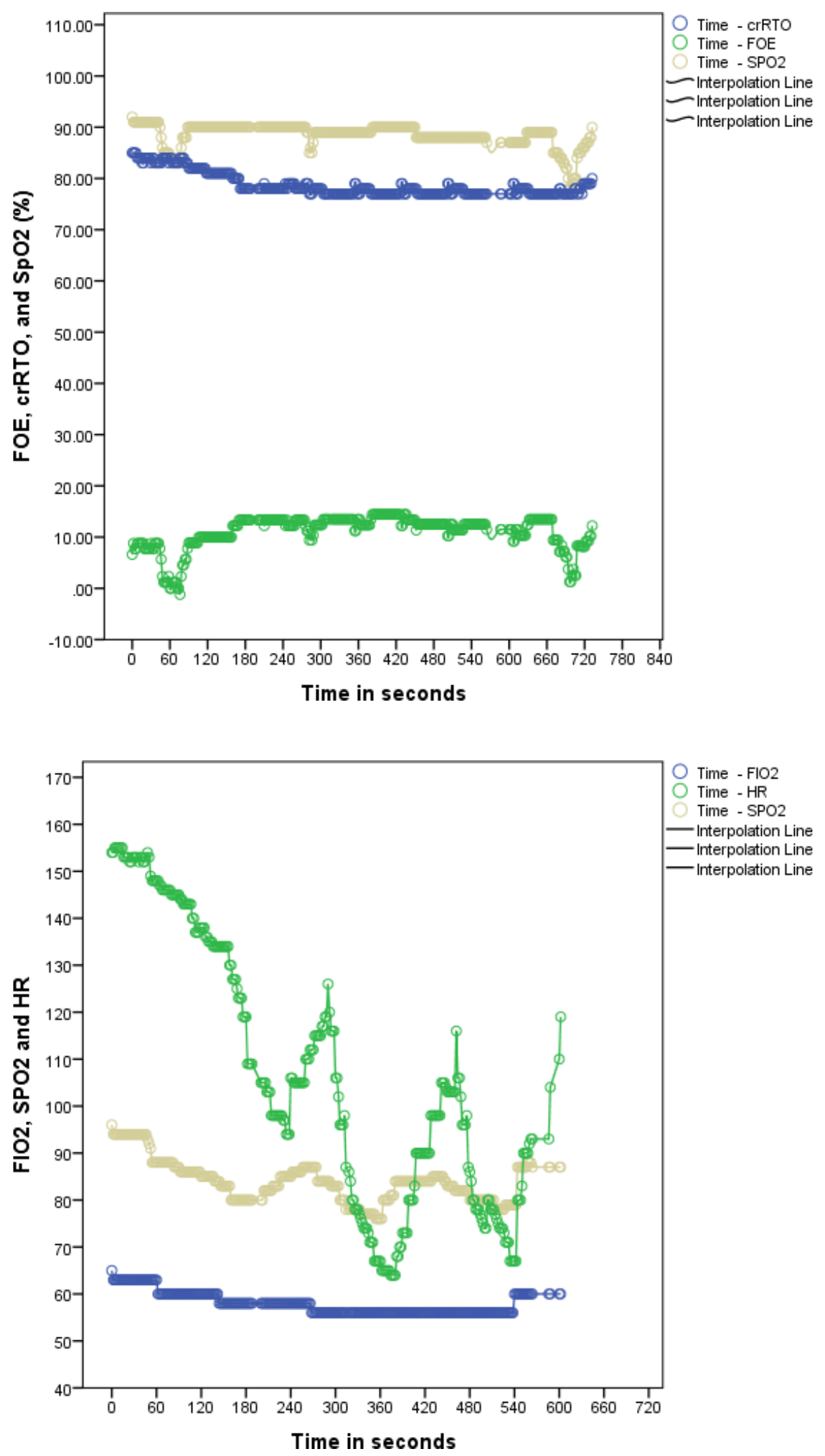


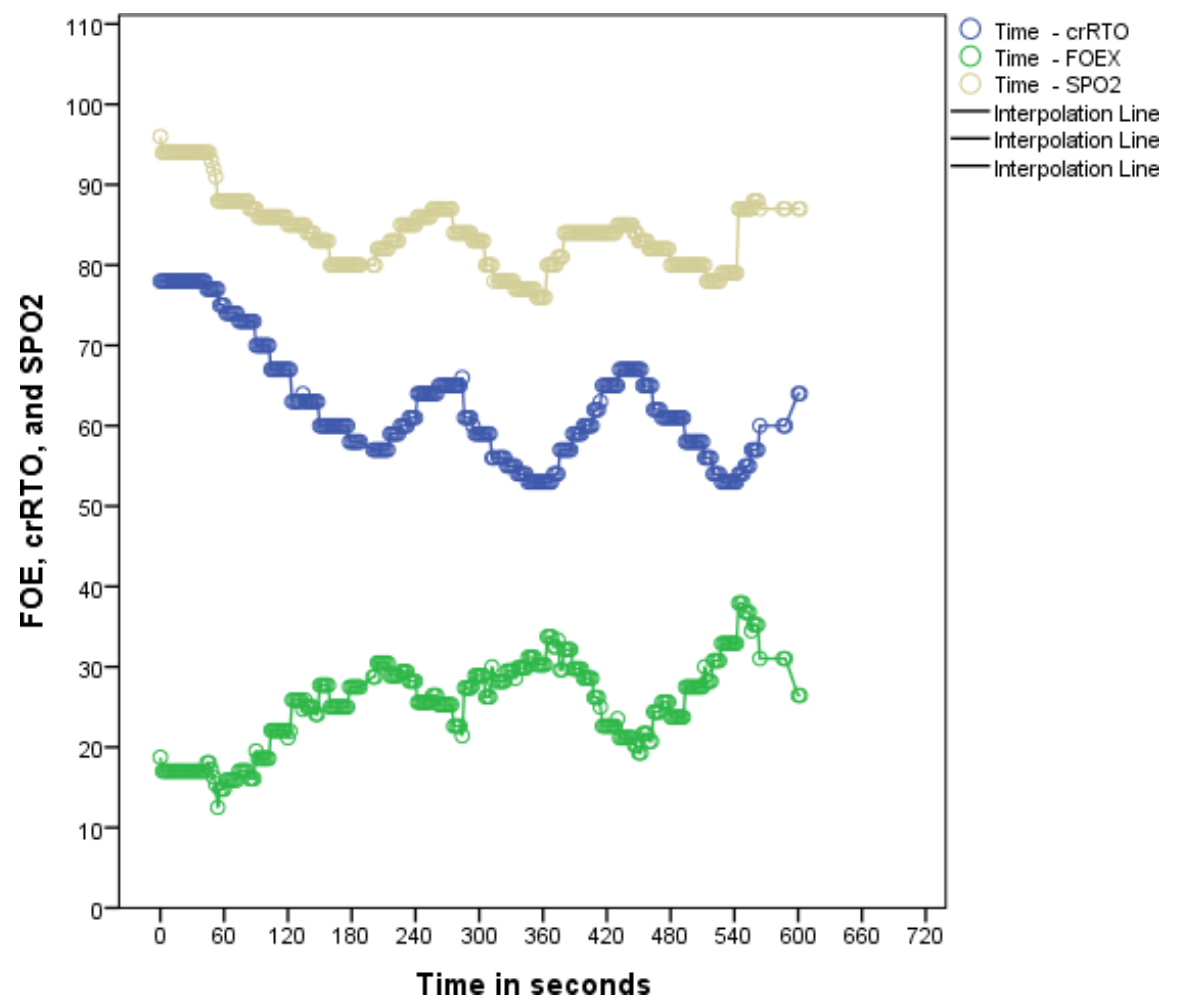

